\title{
Impact of Altered Cell Wall Composition on Saccharification Efficiency in Stem Tissue of Arabidopsis RABA GTPase-Deficient Knockout Mutants
}

\author{
Daniel Lunn • Roger Ibbett • Gregory A. Tucker • \\ Grantley W. Lycett \\ Published online: 13 March 2015 \\ (C) The Author(s) 2015. This article is published with open access at Springerlink.com
}

\begin{abstract}
Use of biomass for second-generation biofuel production is severely hindered by the inherent recalcitrance of the plant cell wall to digestion. Trafficking is crucial for compartmentalisation within the cell. This process is partly regulated by small Rab GTPase proteins. In particular, control of trafficking to the cell wall is regulated through the RABA clade. Manipulation of this regulatory system offers tantalising opportunities for manipulation of cell wall composition and hence recalcitrance. Trafficking-defective $r a b A$ mutants have already been shown to impact cell wall composition. To study the impacts of these mutants on cell wall digestion, we developed a saccharification process for Arabidopsis based on the hot water method. We then showed that following pre-treatment, stems from the T-DNA knockouts of the three $R A B A 4$ genes expressed in Arabidopsis stem show an increased sugar release on saccharification. These rabA4 mutants have been shown to impact the "hemicellulose-rich" fraction during cell wall fractionation. Furthermore, we go on to show that these mutant lines also show increased sugar
\end{abstract}

D. Lunn $\cdot$ R. Ibbett $\cdot$ G. A. Tucker $\cdot$ G. W. Lycett $(\bowtie)$

School of Biosciences, University of Nottingham, Sutton Bonington

Campus, Loughborough LE12 5RD, UK

e-mail: grantley.lycett@nottingham.ac.uk

D. Lunn

e-mail: daniel.lunn@wsu.edu

R. Ibbett

e-mail: roger.ibbett@nottingham.ac.uk

G. A. Tucker

e-mail: gregory.tucker@nottingham.ac.uk

Present Address:

D. Lunn

Institute of Biological Chemistry, Washington State University,

Pullman, WA 99163, USA release when subjected to saccharification without pre-treatment. Finally, we used X-ray diffraction to show that rabA4 mutants had no impact on cellulose crystallinity, thus supporting the hypothesis that the increases in saccharification were not due to alterations of the cellulose microfibrils but were a direct effect of reduced hemicellulose levels. We also present data to show that the growth characteristics of these plants were unaffected. The data obtained from these lines are most easily explained by the reported alteration in hemicellulose increasing pre-treatment efficiency.

Keywords Rab GTPase · Vesicle trafficking · Cell wall · Biofuels $\cdot$ Saccharification $\cdot$ Hemicellulose

\section{Introduction}

An increasing demand globally for finite fossil fuels and concern over greenhouse gas emissions from their combustion has justified an increase in bioenergy research. Traditionally, bioenergy was considered expensive and uneconomic; however, an ever-rising oil price has redressed the balance in favour of bioenergy [1]. Mounting concerns about the sustainability of bioenergy crops and food security have focused attention on using non-food biomass. These secondgeneration feed stocks employ lignocellulosic biomass to create biofuel [2]. However, the cell wall has evolved to defend the organism from animal, viral, fungal and bacterial attack [3] and, as a result, requires pre-treatment before the hydrolysis step in the production process [4]. Due to this requirement, two solutions have been explored through the literature. The first of these focuses on efficiency of pre-treatment, and the second focuses on exploration of genetic modification to 
increase susceptibility to pre-treatment and/or enzymatic hydrolysis. Currently, in the literature, various types of pretreatment for Arabidopsis have been described, which tend to use treatment with acids, alkalis and hot water [5-8], and a number of protocols for high-throughput analysis have also been described $[9,10]$. However, a consensus throughout the community has yet to be established. The second line of research has been the exploration of gene knockouts which could increase the susceptibility to enzyme hydrolysis. Most of the work in this field has focused on genes affecting lignin and xylan composition [6-8, 11-13]. However, direct interference with cell wall biosynthesis machinery could have an adverse effect on the agronomic properties of the crop. Due to this, knockout or knockdown approaches to inhibiting genes encoding components of the trafficking machinery may be a viable alternative, as they often exhibit a high degree of redundancy within their gene families. In eukaryotes, Rab GTPases regulate vesicle trafficking, and in Arabidopsis, 57 homologues have been identified and grouped into clades (RABA, RABB, RABC, etc.) based upon sequence homology [14]. The RABA clade has 26 members and has been further divided into subclades (RABA1, RABA2, RABA3, etc.) with individual proteins identified by lowercase letters (RABA1a, RABA1b, etc.). Members of the RABA clade regulate trafficking to the cell wall through the trans-Golgi network (TGN), by acting as molecular switches for vesicle docking [15]. In Arabidopsis, RABA2 and RABA3 proteins have been associated with trafficking to the cell plate [16], and in tomato, inhibition of a $R A B A 1$ orthologue has been shown to impact pectin levels in fruit [17]. More recently, three independent lines with T-DNA knockouts in the three different $R A B A 1$ genes (RABAla, RABAlc and RABAld) that are expressed in stem tissue were tested and each showed similar levels of reduction in the level of pectin. Similarly, consistent results were obtained for reductions in the cellulose and hemicellulose fractions in different $r a b A 2$ and $r a b A 4$ mutant lines, respectively [18]. What is particularly interesting from the work carried out by Lunn and colleagues [18] is that the gene knockouts described in that study impacted on cell wall composition without directly affecting cell wall biosynthetic machinery. Here, we show that rabA4 knockout mutants exhibited an increased sugar release upon saccharification, both with and without pre-treatment.

\section{Materials and Methods}

Plant Material

Arabidopsis thaliana Col-1 and mutant lines are listed in Table 1. Each of the genes expressed in stem tissue of Arabidopsis was identified, and one T-DNA knockout mutant line was used for each gene. The characterisation of these lines
Table 1 List of $r a b A$ mutant lines used

\begin{tabular}{lll}
\hline $\begin{array}{l}\text { RAB gene in which T-DNA } \\
\text { insert occurs }^{\mathrm{a}}\end{array}$ & $\begin{array}{l}\text { AGI ID no. for } \\
\text { gene }\end{array}$ & $\begin{array}{l}\text { NASC ID no. for } \\
\text { Arabidopsis line }\end{array}$ \\
\hline RABA1a & At1g06400 & N653306 \\
RABA1c & At5g45750 & N679206 \\
RABA1d & At4g18800 & N588879 \\
$R A B A 1 i$ & At1g28550 & N810111 \\
$R A B A 2 b$ & At1g07410 & N806084 \\
$R A B A 2 d$ & At5g59150 & N668131 \\
$R A B A 3$ & At1g01200 & N813511 \\
$R A B A 4 a$ & At5g65270 & N656868 \\
$R A B A 4 b$ & At4g39990 & N659845 \\
$R A B A 4 e$ & At2g22390 & N620411 \\
\hline
\end{tabular}

${ }^{\text {a }}$ Gene nomenclature according to Rutherford and Moore [14]

and the confirmation of the lack of an RNA transcript has been described previously [18].

\section{Arabidopsis Growth and Phenotyping}

Plants were grown under glass in the summer. Glasshouse conditions were as follows: $22^{\circ} \mathrm{C}$ with 16 -h light and 8-h dark period, light intensity of $150 \mu \mathrm{mol} / \mathrm{m}^{2} / \mathrm{s}$. For compositional and digestibility assays, 50 plants of each line were placed in a randomised block structure. Stem material from the 50 plants was pooled at the senescent stage for analysis. Plants were grown in three successive months and treated as triplicates. Phenotype analysis was conducted on three replicates grown on separate occasions, using a randomised block structure, with ten plants each comprising one replicate. Phenotypic analysis was carried out based on the methodology described by Boyes et al. [19].

\section{Biomass Pre-Treatment}

Dry, senescent Arabidopsis stem samples were milled to a particle size of $700 \mu \mathrm{m}$. Milled Arabidopsis stem (250 mg) was placed in a sealed glass reaction vial. Samples were placed into an Anton Parr Monowave 300 reactor, programmed for maximum heating rate, and incubated at $121{ }^{\circ} \mathrm{C}$ for 10,20 or $30 \mathrm{~min}$. Three separate treatments"dry", $\mathrm{dH}_{2} \mathrm{O}$ and $1 \% \mathrm{H}_{2} \mathrm{SO}_{4}$-were employed. The last two, at 1:10 v/w, were employed to assess the best pre-treatment. The liquor produced from the reaction was removed by filtration and discarded. Residues from the pre-treatments were suspended in $2.5 \mathrm{ml}$ of $\mathrm{dH}_{2} \mathrm{O}$ and adjusted to $\mathrm{pH} 5.4$ using $2 \mathrm{M} \mathrm{NaOH}$. The residue was then placed on aluminium foil and dried overnight in an oven at $60{ }^{\circ} \mathrm{C}$. Samples were stored in an air-tight plastic bag at room temperature until required for the digestion assay. 


\section{Glucose Measurements}

Milled stem or pre-treated residue $(30 \mathrm{mg}$ ) was subjected to a two-stage acid hydrolysis. Twelve molar sulphuric acid ( $1 \mathrm{ml})$ was added, and the sample was incubated for $1 \mathrm{~h}$ at $37^{\circ} \mathrm{C}$ then

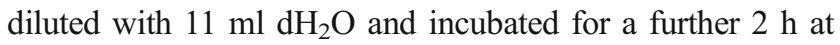
$100{ }^{\circ} \mathrm{C}$. The glucose content of the supernatant was determined by high-performance anion exchange chromatography with pulsed amperometric detection (HPAEC-PAD) (Dionex, UK) using a CarboPac PA20 column with a $50 \mathrm{mM} \mathrm{NaOH}$ isocratic system and flow rate of $0.5 \mathrm{ml} / \mathrm{min}$ at $30{ }^{\circ} \mathrm{C}$. Glucose was used to generate a standard curve with mannitol as internal standard.

\section{Saccharification Assay}

A cellulase stock solution (Trichoderma reesei from SigmaAldrich) was made at a concentration of $10 \mathrm{mg} / \mathrm{ml}$ in $50 \mathrm{mM}$ $\mathrm{Na}$ citrate buffer at $\mathrm{pH}$ 6.5. The enzyme was dialysed against 21 of the $50 \mathrm{mM} \mathrm{Na}$ citrate buffer overnight at $4{ }^{\circ} \mathrm{C}$. Pretreated Arabidopsis milled stem (100 mg) was weighed out, and a 1-ml aliquot of the stock cellulase enzyme was added to each sample. The samples were mixed and made up to $10 \mathrm{ml}$ with $50 \mathrm{mM}$ Na citrate buffer. Samples were then incubated at $55^{\circ} \mathrm{C}$ using an orbital shaker at $150 \mathrm{rpm}$. Aliquots were taken of the sample at time points $0,2,4,6$ and $24 \mathrm{~h}$ and glucose released into solution determined by HPAEC-PAD as described above.

\section{Lignin Assay}

Lignin was quantified directly using a modified version of the acetyl bromide method. Standards were made using $10 \mathrm{mg}$ of lignin (Sigma batch no. 04414PE) dissolved in $80 \%$ dioxane. Aliquots were made ranging from 0.2 to $0.6 \mathrm{ml}$ in increments of $0.1 \mathrm{ml}$. Acetyl bromide $(0.5 \mathrm{ml}, 25 \%)$ was added and incubated at $50{ }^{\circ} \mathrm{C}$ for $30 \mathrm{~min}$. Samples were allowed to cool, and then, $2.5 \mathrm{ml}$ acetic acid (25\%), $1.5 \mathrm{ml} \mathrm{NaOH}(0.3 \mathrm{M})$ and $0.5 \mathrm{ml}$ hydroxylamine $\mathrm{HCl}(0.5 \mathrm{M})$ were added, and the volume was made up to $10 \mathrm{ml}$ with acetic acid (100\%). Milled Arabidopsis stem (particle size $700 \mu \mathrm{m}$ ) was weighed out to $100 \mathrm{mg}$, and $4 \mathrm{ml}$ acetyl bromide (25\%) added. Samples were incubated for $3 \mathrm{~h}$ at $50{ }^{\circ} \mathrm{C}$. Cooled samples were made up to $16 \mathrm{ml}$ with acetic acid (100\%). An aliquot of $0.5 \mathrm{ml}$ of sample was taken, and $2.5 \mathrm{ml}$ acetic acid, $1.5 \mathrm{ml} \mathrm{NaOH}(0.3 \mathrm{M}), 0.5 \mathrm{ml}$ hydroxylamine $\mathrm{HCl}$ $(0.5 \mathrm{M})$ and $5 \mathrm{ml}$ of acetic acid $(100 \%)$ were added to give a final volume of $10 \mathrm{ml}$. Blank, standard and samples were read using a scanning UV spectrophotometer through the wavelengths of 250-400 nm with lignin reading taken at $280 \mathrm{~nm}$.
Determination of Cellulose Crystallinity

Milled Arabidopsis stem samples (particle size $700 \mu \mathrm{m}$ ) were measured in reflection mode, using a vertical $\theta-\theta$ diffractometer (D5000, Siemens/Bruker GmbH). Irradiation was from a copper $\mathrm{K} \alpha \mathrm{X}$-ray source $(\lambda=1.541 \AA)$, with static slit optics at source and detector. Samples were loaded into circulator holders with light pressure, which were rotated about the phi-axis during measurement. $2 \theta$ Scans were performed from $5^{\circ}$ to $50^{\circ}$, in $0.05^{\circ}$ increments. Intensities were background corrected by linear subtraction between $5^{\circ}$ and $50^{\circ} 2 \theta$. Crystalline diffracted intensities were determined by subtraction of an experimental amorphous profile of a highly ball milled reference stem material from each of the sample diffraction profiles. The total crystallinity of each sample was calculated as the proportion of crystalline integrated intensity against the total integrated intensity. An estimate of the fibril lateral dimension $(d)$ was obtained by measurement of the width of the 200 reflection of cellulose I $\left(2 \theta=22.5^{\circ}\right)$, according to the Scherrer equation $(d=0.9 \lambda / \mathrm{IB} \cos \theta)$. Corrections for instrumental and defect broadening were not deemed necessary due to the breadth of this peak. The integral breadth (IB) is defined as the integrated intensity of the fitted peak divided by the peak height, where $\lambda$ is the $\mathrm{X}$-ray wavelength $(0.1541 \mathrm{~nm})$ and $\theta$ is the diffraction angle. The constant 0.9 was selected for consistency with published data [20].

\section{Statistics}

Statistical analysis for the saccharification analysis was performed by $t$ test comparing the wild type against the knockout lines at individual time points, over the time course of analysis. Each replicate represents 50 plants pooled together, with three replicates performed overall. Analysis of the phenotypic data was conducted using ANOVA to distinguish significance between the lines. The data comprised three separate replicates each consisting of measurements for ten individual plants.

\section{Results}

\section{Pre-Treatment Selection}

In order to assess whether or not these modifications impacted cell wall recalcitrance, we set out to identify a suboptimal pretreatment regime that results in the release of around $50 \%$ of the available glucose. The literature describes a wide variety of pre-treatment methods. In this study, we tested three pretreatment methods: dry (biomass with residual moisture), $\mathrm{dH}_{2} \mathrm{O}$ and $1 \% \mathrm{H}_{2} \mathrm{SO}_{4}$, over three exposure times 10, 20 and $30 \mathrm{~min}$, in a microwave reactor set at $121^{\circ} \mathrm{C}$. The resultant 
residues were then subjected to a standard enzyme hydrolysis. The results of these experiments are shown in Fig. 1.

The percentage of the available glucose released from the untreated biomass was around $10 \%$ (Fig. 4). With a dry pretreatment, this was increased to around $30 \%$ after 10-min exposure and to around $40 \%$ after 20- and 30-min exposure. At this point, it is worth noting that the senescent stem of Arabidopsis had an average moisture content of around $15 \%$ (data not shown). Pre-treatment with $\mathrm{dH}_{2} \mathrm{O}$ yielded a
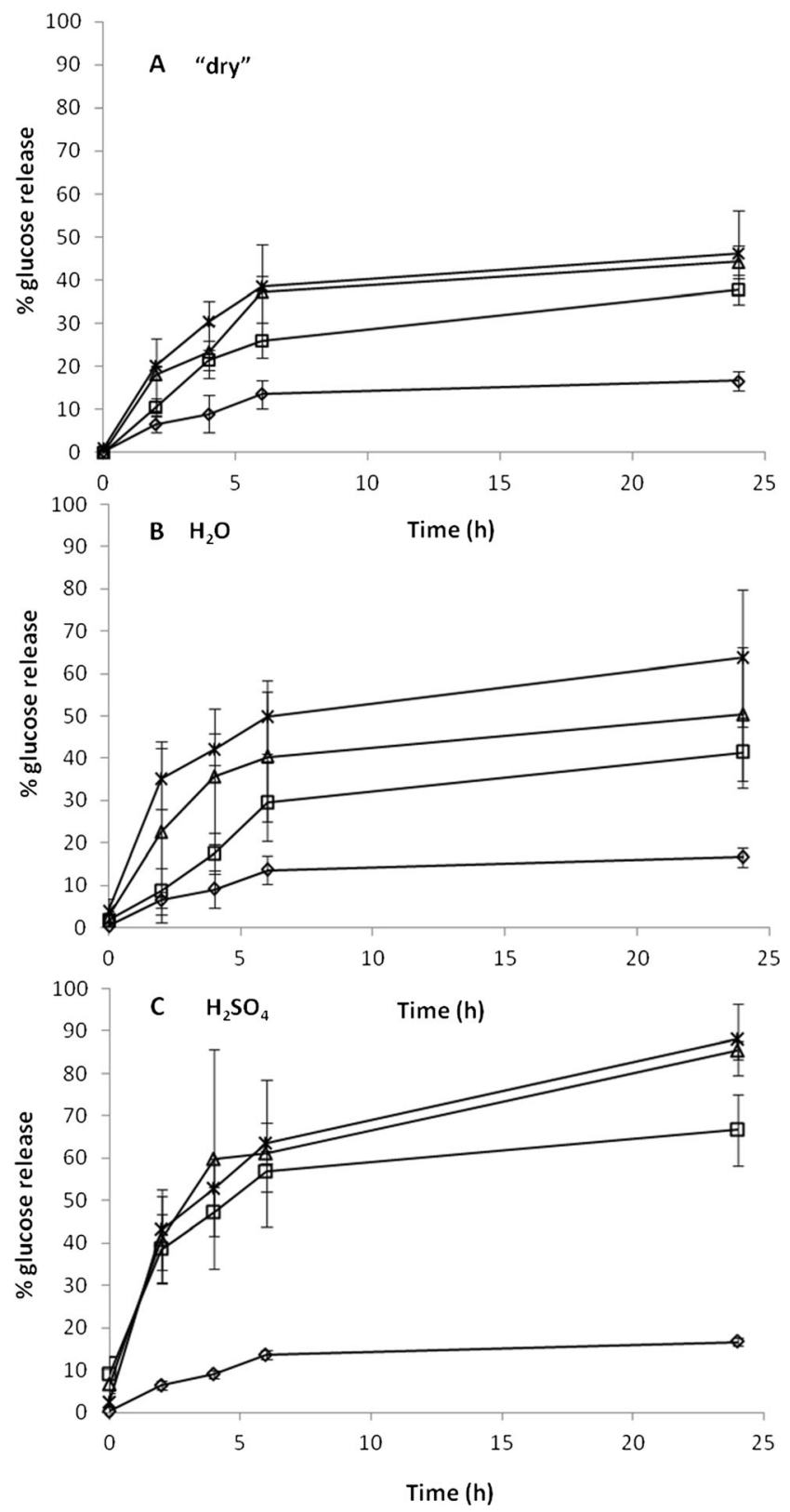

Fig. 1 Effect of pre-treatment conditions on glucose release from wildtype Arabidopsis stem. Milled stem tissue from wild-type plants was sub-

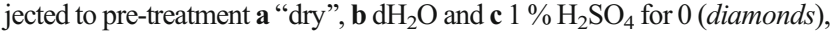
10 (squares), 20 (triangles) or $30 \mathrm{~min}$ (crosses). The resultant residue was then subjected to a standard enzyme hydrolysis and the percentage available glucose released monitored over $24 \mathrm{~h}$. Data points show the mean with error bars showing standard deviation $(n=3)$ percentage glucose release of around 30, 50 and $65 \%$ after 10-, 20- and 30-min exposure, respectively. Treatment with $1 \% \mathrm{H}_{2} \mathrm{SO}_{4}$ again increased percentage glucose release to around 65,85 and $90 \%$ of the total available after 10, 20 and $30 \mathrm{~min}$ of exposure.

Impact of $r a b A$ Knockouts on Cell Wall Recalcitrance

Data in Fig. 1 show that a pre-treatment in $\mathrm{dH}_{2} \mathrm{O}$ for 20 min yielded $50 \%$ total glucose release after enzyme hydrolysis. This pre-treatment was therefore selected as a standard method to screen all $r a b A$ knockouts (Table 1) and wild-type plants. The Arabidopsis knockout lines with T-DNA inserts in individual $R A B A$ genes, used in this study, have been described previously [18]. Milled stem tissue was subjected to this standard pre-treatment and the resultant residues analysed for total glucose content. The results are shown in Fig. 2. The residues all contained between 80 and $90 \%$ glucose, and there were no significant differences between the wild type and any of the knockout lines. All the residues were then subjected to a standard enzyme saccharification assay and the percentage available glucose released was recorded over time (Fig. 3). The average percentage glucose release for $r a b A 1$ gene knockouts was identical to that for the wild type at around $50 \%$. A similar result was obtained with the $r a b A 2$ knockouts. In this case, there was a slight increase in the rate of release, compared to the wild type, but this was not statistically significant. The rabA3 knockout showed a slight increase in recalcitrance with a final percentage glucose release of around $40 \%$ which was significantly different from the wild-type plants $(p=0.02)$. Finally, the rabA4 knockouts all had levels of glucose release that were, compared to the wild type, statistically significantly increased, to a final yield of between 70 and $80 \%(p \leq 0.001)$.

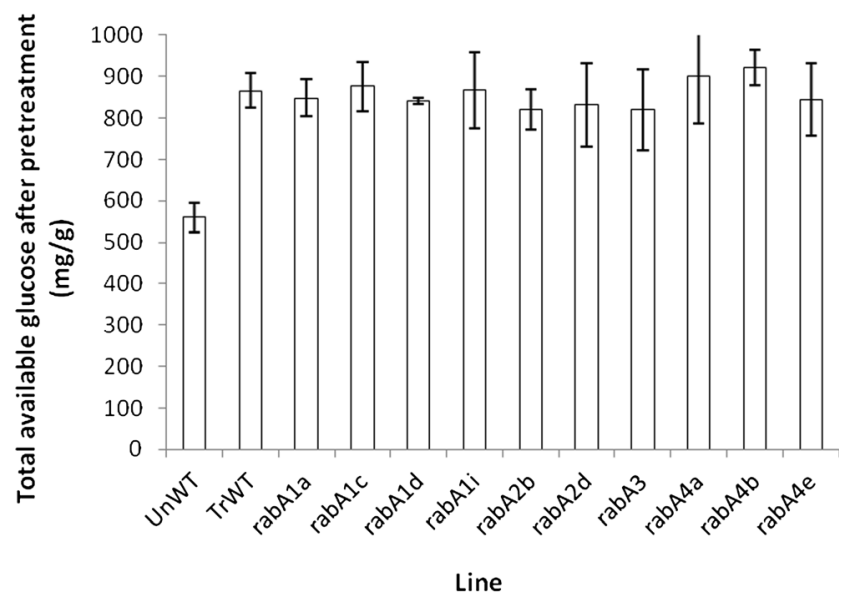

Fig. 2 Glucose content of pre-treated residues from wild type and $r a b A$ knockout lines. Stem tissues from wild type and $r a b A$ knockouts were pre-treated at $121{ }^{\circ} \mathrm{C}$ for $20 \mathrm{~min}$ in $\mathrm{dH}_{2} \mathrm{O}$. The residues were then dried and glucose content analysed. Results are expressed as milligrams of glucose per gram of residue. Error bars show standard deviation $(n=3)$ 

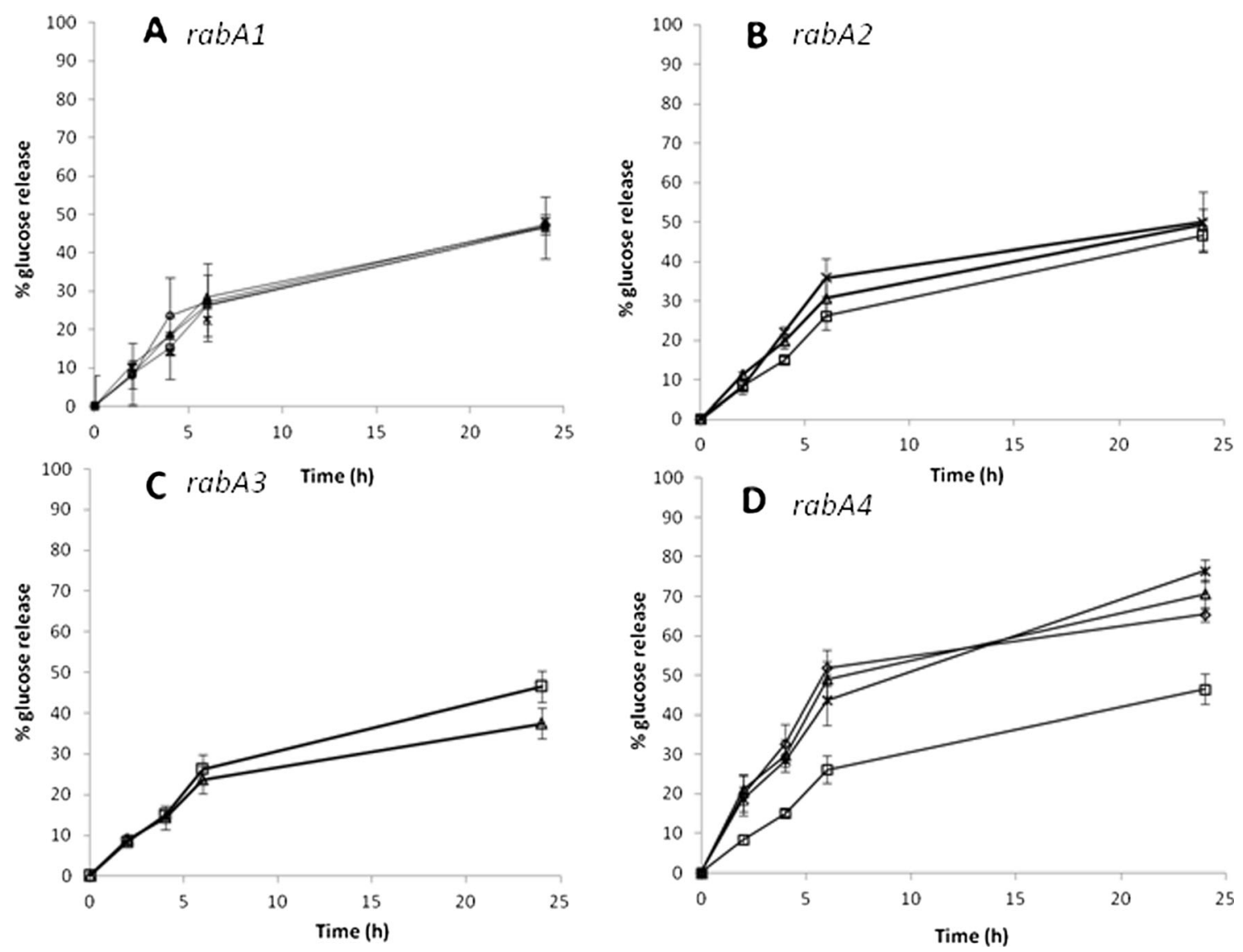

Fig. 3 Saccharification of wild type and pre-treated $r a b A$ knockouts. Dried residues from pre-treated wild type and knockout lines stem tissue were subjected to a standard enzyme hydrolysis, and glucose released was monitored over $24 \mathrm{~h}$. On all graphs, treated wild type is shown by squares. Key; a RABAl subclade: rabAla (triangles), rabAlc (crosses),

\section{Recalcitrance of Untreated rabA4 Knockouts}

The rabA4 mutants all demonstrated a statistically significant increase in glucose release following pre-treatment. The next step was to examine to what extent, if any, recalcitrance may have been impacted in this tissue without any pre-treatment. To do this, wild type and all three rabA4 knockouts were milled and subjected to the same saccharification assay but without any pre-treatment; the data are shown in Fig. 4. For wild-type stem tissue, around $10 \%$ of the total available glucose was released after $24 \mathrm{~h}$. The three rabA4 knockouts, in comparison, resulted in a release of glucose of around $30 \%$ of the total available. This was shown to be significantly different from the wild type $(p<0.001)$.

\section{Impact of Lignin Composition}

In order to exclude the possibility that the altered saccharification shown above was due to reductions in lignin content, we analysed the lignin content of each line relative to the wild type. The results are shown in Fig. 5. The statistically

rabAld (dashes), rabAli (circles); b RABA2 subclade: rabA2b (triangles), rabA2d (crosses); c RABA3 subclade: rabA3 (triangles); d RABA4 subclade: rabA4a (triangles), rabA4b (diamonds), rabA4e (crosses). Error bars show standard deviation $(n=3)$

significant rise in the lignin content of the rabA3 mutant line is consistent with the small increase in recalcitrance of this line. Of the other lines, the rabAli, rabA4a and rabA4e lines showed much smaller but significant increases in lignin levels. None showed any decrease in lignin content.

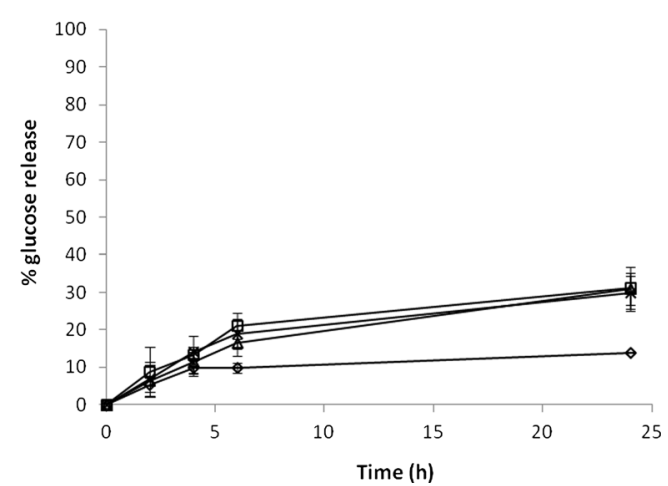

Fig. 4 Saccharification of untreated rabA4 knockouts. Milled stem tissue from wild type and rabA4 knockout lines were subjected to a standard enzyme hydrolysis and glucose released monitored over $24 \mathrm{~h}$. Wild type (diamonds), rabA4a (triangles), rabA4b (crosses), rabA4e (squares). Error bars show standard deviation $(n=3)$ 
Impact of rabA4a on Cellulose Crystallinity

Recalcitrance could be attributed to both the accessibility to the cellulose and enzyme reactivity once associated with the fibril. It was, therefore, interesting to ask the question whether the reduced recalcitrance shown by the $r a b A 4$ knockout mutant lines was associated with any modification to the cellulose crystal structure. Stem tissue from both wild type and $r a b A 4$ knockout lines was analysed using X-ray diffraction. The crystallinity as measured showed a value of $26 \pm 1.5 \%$ for both wild type and rabA4 mutants. To give an actual measure of cellulose crystallinity, this value needs to be corrected for the relative cellulose content of each line. From compositional analysis, this was estimated as being about $40 \%$ for both wild type and rabA4 knockouts, giving a cellulose crystallinity value of around $65 \%$ for each. The lateral dimensions of the cellulose fibrils were estimated to be $2.6 \pm 0.05$ and $2.5 \pm$ $0.05 \mathrm{~nm}$ for wild type and rabA4 lines, respectively.

\section{Impact of rabA4 Knockouts on Growth Properties and Potential Glucose Yield}

One crucial element of all bioenergy themed work is that changes that impact on saccharification might also impact on the growth of the plant, which would imply that the agronomic properties of any crops developed from this technology might be affected. For this reason, an in-depth phenotypic analysis was conducted. This comprised looking at the multiple stages of growth, based on the methods described by Boyes et al. [19]. This analysis showed that there were no gross morphological changes in any of the three $r a b A 4$ knockouts analysed. The major properties of interest, in terms of bioenergy, would be biomass yield, seed yield, root growth and sugar content. The data for these parameters are presented in Fig. 6. After 14 days, the average root length of the wild type was found to be around $5.5 \mathrm{~cm}$ with around 14 lateral roots. The data in
Fig. 6a, b show that there were no statistically significant differences in these root growth parameters between the wild type and any of the mutant lines. The biomass yields, in terms of height and weight of senesced stem tissue "at harvest", are presented in Fig. 6. Wild-type stems averaged about $160 \mathrm{mg}$ with an average length of around $22 \mathrm{~cm}$ per plant, and there were no statistical differences between this and the yield from the rabA4 knockout mutant lines. The production of seed or grain is critical to the second-generation biofuels, if translated to real seed crops; thus, an analysis of seed produced was conducted and is shown in Fig. 6e. Seed yield in wild type was around $380 \mathrm{mg}$ with measurements of the rabA4 lines showing no significant differences. The total glucose in senescent stem material was assessed by direct hydrolysis of stem tissue followed by HPAEC, and the results are shown in Fig. 7. Wild-type plants had a glucose content of around $550 \mathrm{mg} / \mathrm{g}$, and this was statistically similar to the values for all three $r a b A 4$ knockouts. Thus, the knockouts do not demonstrate any significant reduction in glucose yield as compared to wild-type plants.

\section{Discussion}

Arabidopsis is often used as a convenient model to assess the impact of mutations on plant growth and metabolism. In this paper, we have used this model to assess the impact of knockouts in the $R A B A$ gene family on cell wall composition and recalcitrance. From our previously work [18], we have shown that $r a b A 1$ appears to impact the pectic, $r a b A 2$ the cellulosic and rabA4 the hemicellulosic fractions of the wall. In this paper, we extend that observation to assess the impact of these gene knockouts on the recalcitrance of the biomass to saccharification.

Recalcitrance is not an easy parameter to measure directly, and there are a range of reported methods, often dependent on
Fig. 5 Impact of $r a b A$ mutants on lignin composition. Milled stem tissue from wild type and $\mathrm{rabA}$ mutants were subjected to acetyl bromide quantification of lignin composition. Error bars displayed show standard deviation. Statistical analysis was performed by ANOVA $(n=30)$.

The statistical values are as follows: $p<0.001$ (d.f,32 v.r,20.36) with letters annotating groups of means defined by Tukey test

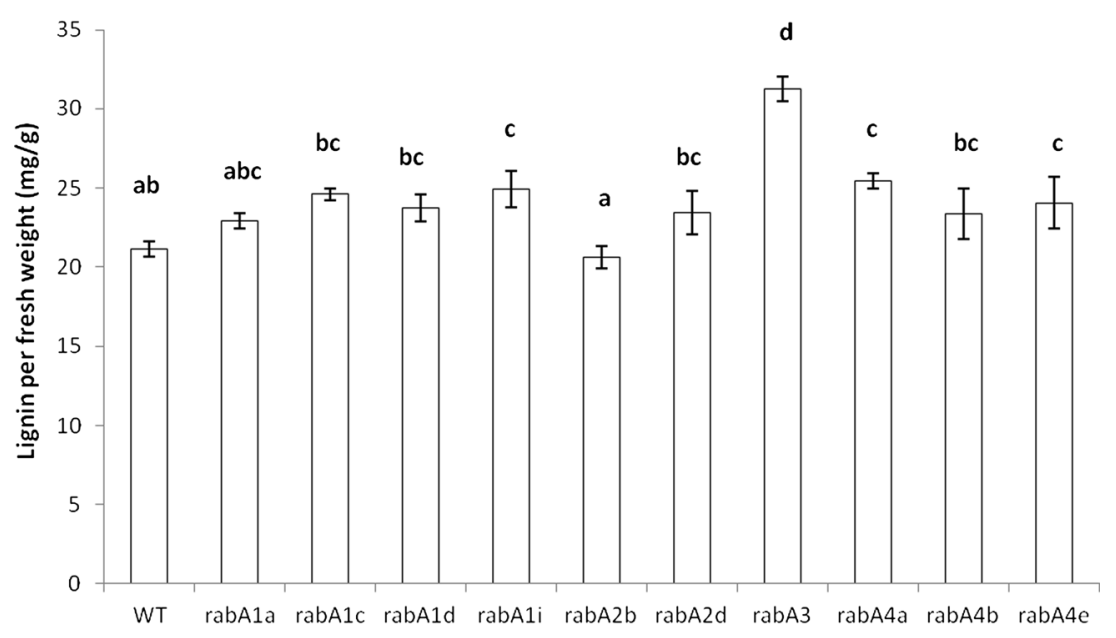

Line 

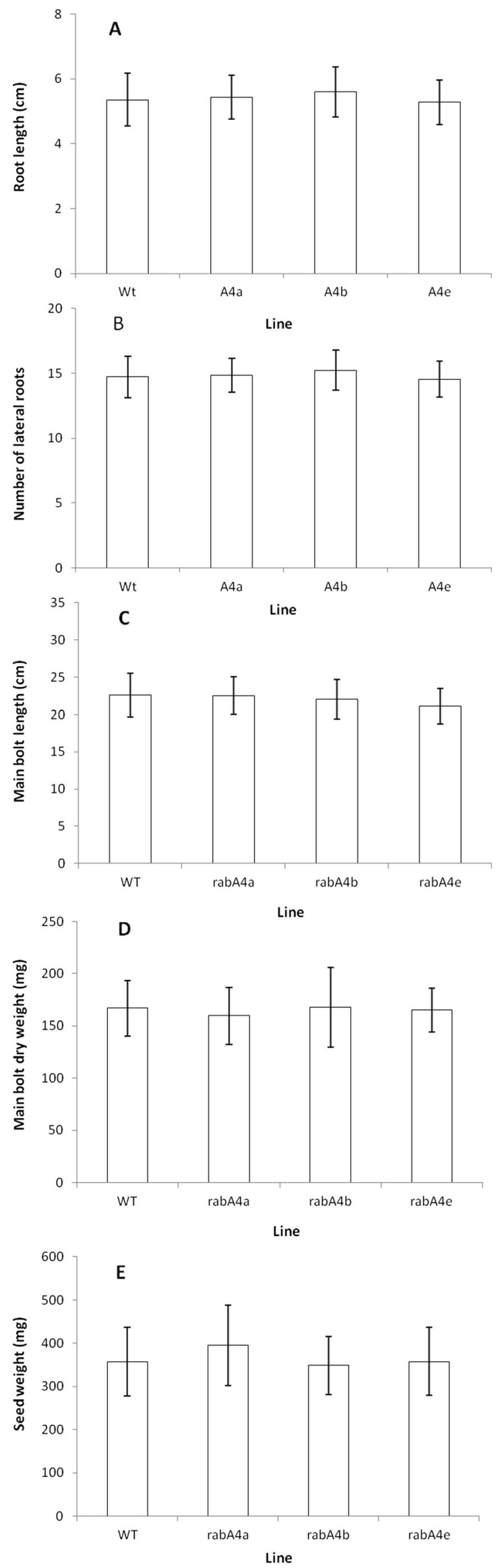

Fig. 6 Phenotypic analysis of rabA4 knockouts. Data show root architecture measurements 14 days post-stratification, biomass yield of senescent stem tissue and seed yield at harvest. Data displayed as root length in centimetres (a), lateral root number (b), stem height in centimetres (c), main bolt dry weight in milligrams (d) and seed weight in milligrams (e). All values are given on a per plant average of 30 biological replicates produced in batches of ten over three growth cycles. Error bars show standard deviation, and statistical analysis was performed by ANOVA $(n=30)$

the biomass being tested. Here, we assessed a range of methods and selected a pre-treatment in $\mathrm{dH}_{2} \mathrm{O}$ at $121^{\circ} \mathrm{C}$ for $20 \mathrm{~min}$. This gave a glucose release of $50 \%$ from wild-type plants. This compares with a similar study which reported a $50 \%$ glucose release using a higher temperature of $200^{\circ} \mathrm{C}$ but with a lower exposure time of $10 \mathrm{~min}$ [6]. This standard pretreatment was then used to assess recalcitrance in stem tissue from wild type and $r a b A$ knockout plants.

The basic compositional analysis carried out previously [18] would suggest that the rabAl knockouts had reduced pectin content in their cell walls. However, this does not appear to have impacted at all on the recalcitrance of the biomass. The role of pectin as a packing agent in the cell wall is well known [21]; however, in the stem material tested, the primary cell wall is less abundant because of secondary thickening. Therefore, pectin is a much reduced component in the material studied. Similarly, the rabA2 knockouts, in which cellulose appears to have been reduced, show no significant effect on recalcitrance. It is, perhaps, not surprising that reducing cellulose would not have an impact on the saccharification. The single rabA3 knockout line appeared to result in an increase in recalcitrance. It is difficult to explain this observation on the basis of carbohydrate polymer composition since this knockout did not appear to be modified in any of the three wall components analysed. However, these are not the only components of the wall that can impact recalcitrance with

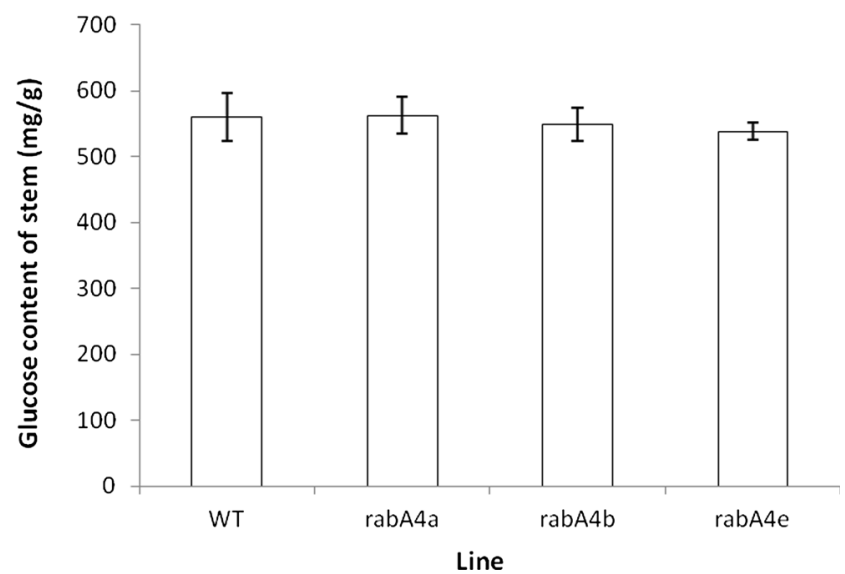

Fig. 7 Glucose content of senescent stem tissue from wild type and rabA4 knockouts. Milled stem tissue was subjected to direct acid hydrolysis and total glucose content monitored by HPAEC. Results are expressed as total glucose per gram of stem tissue. Error bars show standard deviation $(n=3)$ 
lignin an obvious contender as well and lignin levels were slightly raised in this line. Lignin levels in Arabidopsis are quite low, compared to bioenergy crops such as Miscanthus, willow and straw [22-24]; however, it is still possible that modification to this wall fraction might have impacted on recalcitrance.

The most significant effect observed in this paper is the reduction in recalcitrance associated with all three rabA4 knockouts. It appears to be the hemicellulosic fraction of the cell wall component that had been modified in these lines. Although this reduction in hemicellulose appears to result in a slightly increased "purity", and hence content of cellulose, in the dry residue, the magnitude of this increase cannot, on its own, account for the increased glucose release during enzymatic hydrolysis. This would suggest a key role for hemicellulose in determining the level of recalcitrance of the biomass. This is not a novel observation. An increased saccharification in Arabidopsis plants with reduced hemicellulose content has been reported by Brown et al. [12]. In that study, IRX15 knockouts displayed mildly disorganised xylem vessels and decreased xylan content. Two other studies have shown similar results by influencing xylan branching and O-acetylation $[11,13]$. These observations are further supported by studies with wheat straw where extractable mass was correlated with enzyme digestibility of the residue. Upon analysis of these "liquors", hemicelluloses were found to be the predominant component being solubilised [25]. The increase in the proportion of lignin in the rabA4 mutant lines was small and not statistically significant in one line. More importantly, high levels of lignin are known to be associated with increased recalcitrance [26], as seen in the $r a b A 3$ mutant line in this study.

There are perhaps two key components to recalcitrance in biomass. The first is accessibility of the cellulase enzyme to the fibrils and the second the reactivity of that cellulase once it has become associated with the substrate. It was thus possible that the observed results could, in part, be due to changes in the crystal structure of the cellulose fibrils. Cellulose crystallinity is known to influence the digestion profiles of cellulose [27]. In addition, it has been suggested that hemicelluloses are embedded within the cellulose microfibrils [28], and if this is so, reducing the levels of hemicellulose might be expected to have an effect on the cellulose crystallinity. However, there were no differences seen between the rabA4 knockouts and wild type in terms of either crystallinity or fibril diameter. However, it is important to note that these data were taken on "native" stem tissue prior to pre-treatment, and it is thus possible that there are differential changes in crystal structure occurring during the pre-treatment. It is interesting to note that reduced recalcitrance was also evident in native $r a b A 4$ stem tissue without any pre-treatment, suggesting that the modified hemicellulose content may have allowed increased enzyme accessibility even without pre-treatment. However, the glucose release in this case was only $30 \%$ and this would still require a pre-treatment to achieve commercially significant yields. This pre-treatment would however be less severe and thus commercially advantageous. The precise nature of the effect on the hemicellulose fraction in the rabA4 mutants is unknown. This could have resulted in a reduction in the absolute amount of total hemicellulose, a reduction in a specific hemicellulose polymer or indeed in an increased solubility of the hemicellulose, thus reduced recovery in the hemicellulose fraction, perhaps reflecting less effective binding within the cell wall.

Although this study has been carried out in a model plant system, the implications may nonetheless be applicable to commercial bioenergy crops. The RABA family of genes is widespread across a range of plant phyla including wheat, rice and maize $[29,30]$. Thus, lines in which activities of the $R A B A 4$ orthologues in these species have been inhibited may have considerable commercial relevance, especially considering the additional fact that these genetic modifications do not appear to adversely impact on the yield of biomass or its total glucose content.

Acknowledgments The research reported here was supported (in full or in part) by a Biotechnology and Biological Sciences Research Council (BBSRC) Targeted Priority Studentship in Bioenergy (number BB/ G017964) to DL and the BBSRC Sustainable Bioenergy Centre (BSBEC), under the programme for Lignocellulosic Conversion To Ethanol (LACE) [Grant Ref. BB/G01616X/1]. This is a large interdisciplinary programme, and the views expressed in this paper are those of the authors alone and do not necessarily reflect the views of the collaborators or the policies of the funding bodies.

Authors' Contributions RI collected and analysed the crystallinity data; all other data collection and analysis was conducted by DL. The project was conceived and directed by GWL and GAT. All authors contributed to the writing of the manuscript and approved the final version.

Conflict of Interest The authors declare no competing interests

Open Access This article is distributed under the terms of the Creative Commons Attribution License which permits any use, distribution, and reproduction in any medium, provided the original author(s) and the source are credited.

\section{References}

1. Pauly M, Keegstra K (2008) Cell wall carbohydrates and their modifications as a resource for biofuels. Plant J 54:559-568

2. Yuan JS, Tiller KH, Al-Ahmad H, Stewart NR, Stewart CN Jr (2008) Plant to power: bioenergy to fuel the future. Trends Plant Sci 13:421429

3. Sarkar P, Bosneaga E, Auer M (2009) Plant cell walls throughout evolution. Towards a molecular understanding of their design principles. J Exp Bot 60:3615-3635

4. McCann MC, Carpita NC (2008) Designing the deconstruction of plant cell walls. Curr Opin Plant Biol 11:314-320 
5. Lionetti V, Francocci F, Ferrari S, Volpi C, Bellincampi D, Galletti R, D’Ovidio R, De Lorenzo G, Cervone F (2009) Engineering the cell wall by reducing de-methyl-esterified homogalacturonan improves saccharification of plant tissues for bioconversion. Proc Natl Acad Sci U S A 107:616-621

6. Li X, Ximenes E, Kim Y, Slininger M, Meilan R, Ladisch M, Chapple C (2010) Lignin monomer composition affects Arabidopsis cell-wall degradability after liquid hot water pre-treatment. Biotechnol Biofuels 3:27-34

7. Eudes A, George A, Mukerjee P, Kim J, Pollet B, Benke P, Yang F, Mitra P, Sun L, etinkol , Chabout S, Mouille G, Soubigou-Taconnat L, Balzergue S, Singh S, Holmes B, Mukhopadhyay A, Keasling J, Simmons B, Lapierre C, Ralph J, Loqué D (2012) Biosynthesis and incorporation of side-chain-truncated lignin monomers to reduce polymerization and enhance saccharification. Plant Biotechnol 10:609620

8. Van Acker R, Vanholme R, Storme V, Mortimer J, Dupree P, Boerjan W (2013) Lignin biosynthesis perturbations affect secondary cell wall composition and saccharification yield in Arabidopsis thaliana. Biotechnol Biofuels 6:46-63

9. Gomez L, Whitehead C, Barakate A, Halpin C, McQueen-Mason S (2010) Automated saccharification assay for determination of digestibility in plant materials. Biotechnol Biofuels 3:23-35

10. Santoro N, Cantu SL, Tornqvist C-E, Falbel TG, Bolivar JL, Patterson SE, Pauly M, Walton JD (2010) A high throughput platform for screening milligram quantities of plant biomass for lignocellulose digestibility. Bioenergy Res 3:93-102

11. Mortimer J, Miles G, Brown M, Zhang Z, Segura M, Weimar T, Yu X, Seffen K, Stephens E, Turner S, Dupree P (2010) Absence of branches from xylan in Arabidopsis gux mutants reveals potential for simplification of lignocellulosic biomass. Proc Natl Acad Sci U S A 107:17409-17414

12. Brown DE, Wightman R, Zhang Z, Gomez LD, Atanassov I, Tryfona T, McQueen-Mason SJ, Dupree P, Turner S (2011) Arabidopsis genes IRREGULAR XYLEM (IRX15) and IRX15L encode DUF579containing proteins that are essential for normal xylan deposition in the secondary cell wall. Plant J 66:401-413

13. Xiong G, Cheung K, Pauly M (2013) Xylan O-acetylation impacts xylem development and enzymatic recalcitrance as indicated by the Arabidopsis mutant tbl29. Mol Plant 6:1373-1375

14. Rutherford S, Moore I (2002) The Arabidopsis Rab GTPase family: another enigma variation. Curr Opin Plant Biol 5:518-528

15. Lycett G (2008) The role of Rab GTPases in cell wall metabolism. J Exp Bot 59:4061-4074

16. Chow CM, Neto H, Foucart C, Moore I (2008) Rab-A2 and Rab-A3 GTPases define a trans-Golgi endosomal membrane domain in Arabidopsis that contributes substantially to the cell plate. Plant Cell 20:101-123
17. Lunn D, Phan TD, Tucker GA, Lycett GW (2013) Cell wall composition of tomato fruit changes during development and inhibition of vesicle trafficking is associated with reduced pectin levels and reduced softening. Plant Physiol Biochem 66:91-97

18. Lunn D, Gaddipati SR, Tucker GA, Lycett GW (2013) Null mutants of individual RABA genes impact the proportion of different cell wall components in stem tissue of Arabidopsis thaliana. PLoS ONE 8: e75724

19. Boyes DC, Zayed AM, Ascenzi R, McCaskill AJ, Hoffman NE, Davis KR, G rlach J (2001) Growth stage-based phenotypic analysis of Arabidopsis: a model for high throughput functional genomics in plants. Plant Cell 13:1499-1510

20. Kennedy CJ, Cameron GJ, Turcova AS, Apperley DC, Altaner C, Wess TJ, Jarvis MC (2007) Microfibril diameter in celery collenchyma cellulose: X-ray scattering and NMR evidence. Cellulose 14:235246

21. Harholt J, Suttangkakul A, Scheller HV (2010) Biosynthesis of pectin. Plant Physiol 153:384-395

22. Yashoda M, Liu Y, Uchida S, Kawarada K, Ukagami Y, Ichinose H, Kaneko S, Fukuda K (2008) Effects of cellulose crystallinity, hemicellulose, and lignin on enzymatic hydrolysis of Miscanthus sinesnsis to monosaccharides. Biosci Biotechnol Biochem 72:805-810

23. Fahmi R, Bridgewater AV, Darvell LI, Jones JM, Yates N, Thain S, Donnison IS (2007) The effect of alkali metals on combustion and pyrolysis of Lolium and Festuca grasses, switchgrass and willow. Fuel 86:1560-1569

24. Buranov AU, Mazza G (2008) Lignin in straw of herbaceous crops. Ind Crop Prod 28:237-259

25. Ibbett R, Gaddipati S, Davies S, Hill S, Tucker G (2011) The mechanisms of hydrothermal deconstruction of lignocellulose: new insights from thermal-analytical and complementary studies. Bioresource Technol 102:9272-9278

26. Limayem A, Ricken SC (2012) Lignocellulosic biomass for bioethanol production: current perspectives and potential issues and future prospects. Prog Energ Combust 38:449-467

27. Hall M, Bansal P, Lee JH, Realff MJ, Bommarius AS (2010) Cellulose crystallinity - a key predictor of the enzymatic hydrolysis rate. FEBS J 227:1571-1582

28. Pauly M, Albersheim P, Darvill A, York WS (1999) Molecular domains of the cellulose/xyloglucan network in the cell walls of higher plants. Plant J 20:629-639

29. Tyler AM, Bhandari DG, Poole M, Napier JA, Jones HD, Lu C, Lycett GW (2015) Gluten quality of bread wheat is associated with activity of RabD GTPases. Plant Biotechnol J 13:163-176

30. Zhang J, Hill DR, Sylvester AW (2007) Diversification of the RAB guanosine triphosphate family in dicots and monocots. J Integrative Plant Biol 49:1129-1141 